\title{
The short hyperacusis questionnaire: A tool for the identification and measurement of hyperacusis in the Italian tinnitus population
}

\author{
Federica Tortorella, ${ }^{1}$ Silva Pavaci, ${ }^{1}$ Alessandra Barbara Fioretti, ${ }^{2}$ Francesco Masedu, ${ }^{1}$ \\ Maria Lauriello, ${ }^{1}$ Alberto Eibenstein ${ }^{1,2}$
}

${ }^{1}$ Department of Applied Clinical Sciences and Biotechnology, L'Aquila University, L'Aquila;

${ }^{2}$ Tinnitus Center, European Hospital, Rome, Italy

\begin{abstract}
The aim of this study was to explore the collapsibility of the Italian version of Khalfa's hyperacusis questionnaire (HQ). We identified the more statistically significant items of the HQ and created the short hyperacusis questionnaire (SHQ). We recruited 117 consecutive outpatients with a primary complaint of tinnitus at least from 3 months. All patients filled in the complete Italian version of the HQ and underwent an audiological examination including uncomfortable loudness levels (ULLs). A logistic model was carried out getting odds ratios (ORs) estimates of hyperacusis according to the items responses. To create the SHQ, we selected six items that were the only ones to present a statistically significant ORs value different from 1 . The internal consistency of the SHQ was assessed by means of Cronbach $\alpha$ index. A ROC analysis was performed and an optimal cut-off point was found using the Youden index. Our analysis showed a Cronbach $\alpha$ of 0.67. The area under the ROC curve (AUC), expression of the overall performance of the SHQ versus the ULLs test, was statistically significant $(\mathrm{P}<0.05)$. We found a cut-off of 0.24 as indicative of hyperacusis ( sensitivity $(\mathrm{Se})=78.79 \%$, specificity $(\mathrm{Sp})=42.50 \%)$. SHQ could be useful only in the initial screening of individuals with hyperacusis. We suggest further studies for the validation of a new questionnaire on hyperacusis.
\end{abstract}

\section{Introduction}

Hyperacusis is an auditory hypersensitivity disorder

Correspondence: Federica Tortorella, Department of Applied Clinical Sciences and Biotechnology, L'Aquila University, L'Aquila, Italy.

E-mail: federica.tortorella@gmail.com

Key words: Hyperacusis; questionnaire; tinnitus; uncomfortable loudness levels (ULLs); Khalfa.

Received for publication: 12 May 2017.

Revision received: 12 May 2017

Accepted for publication: 10 August 2017.

This work is licensed under a Creative Commons Attribution NonCommercial 4.0 License (CC BY-NC 4.0).

(C) Copyright F. Tortorella et al., 2017

Licensee PAGEPress, Italy

Audiology Research 2017;7:182

doi:10.4081/audiores.2017.182 characterized by heightened perception of the loudness or annoyance level of sounds. ${ }^{1,2}$ Sounds in the moderate to intense range are perceived as intolerably loud or even painful by patients with hyperacusis. ${ }^{3}$

In 2013, in the first international conference on hyperacusis, two different classifications of this symptom have been proposed in the attempt to find a standardization of terminology. ${ }^{4}$ In his taxonomy, Jastreboff et al. have defined hyperacusis as a negative reaction to a sound, dependent only on its physical characteristics, namely its spectrum and intensity. Differently, misophonia is characterized by negative reactions to a sound with a specific pattern and meaning to an individual patient. In misophonia, the reactions are thought to depend on non-auditory factors, such as the patient's previous evaluation of that sound and the context in which the sound is presented. Jastreboff et al. have also described phonophobia, which is a subset of misophonia, in which fear is the dominant factor. ${ }^{5}$ On the other side, Tyler et al. have suggested the following classification that distinguishes three different type of hyperacusis: loudness hyperacusis, in which some moderately loud sounds are very loud; annoyance hyperacusis, in which some sounds, not always loud, are annoying and fear hyperacusis, when patients are afraid of some sounds, even if they are not loud. ${ }^{6}$ Jastreboff's classification is the current dominant classification and has been in use for a longer period of time, but Tyler's one uses simple terminology and it perhaps more adaptable for a practical use. ${ }^{4}$

The difference of prevalence of hyperacusis found by many authors may depend by the criteria used to define a patient with hyperacusis. In a previous study, it was assessed that hyperacusis affects $8.6 \%$ of the general population and often it co-occurs with tinnitus. This number changed to $19.4 \%$ when examining a subsample of hearing impaired participants. ${ }^{3}$ In another study, hyperacusis, although usually comorbid condition with poor hearing status, was reported to a remarkably high degree of $24.1 \%{ }^{7}$ In a study by Dauman and Bouscau-Faure, the authors assessed both tinnitus and hyperacusis and found that the prevalence of hyperacusis in the tinnitus population was as high as $79 \% .^{2}$ These findings raise the possibility that the two disorders may be linked at some level, so that disruptions in the auditory system, leading to tinnitus, may also lead to hyperacusis. ${ }^{8}$

However, it was observed that even tinnitus patients with clinical normal audiograms can have hyperacusis. ${ }^{1,9}$ In fact, it is possible to have permanent cochlear damage without an elevation of hearing thresholds that is linked to a permanent and progressive degeneration of auditory fibers, which occurs in association with damage of the inner hair cell synapse, such as a loss of synaptic ribbon. ${ }^{10,11}$ This afferent neuronal degeneration seems to be involved in hyperacusis and tinnitus and it suggests that, despite the presence of normal auditory thresholds, the damage to the peripheral hair cell synapse results in changes in the central 
auditory processing. ${ }^{9}$ Weisz et al. confirmed that high frequency tinnitus in patients with normal hearing threshold is triggered by central deafferentation, mostly due to severe inner-hair cell damage which can be very circumscribed. ${ }^{12}$ As proposed by many authors, like Liberman and Kujawa, tinnitus and hyperacusis may be related to a hidden hearing loss in case of a cochlear synaptopathy when the synaptic gain in auditory central circuits is increased for a diminished neural signal from a damaged cochlea. ${ }^{13}$

Nowadays the central origin of tinnitus and hyperacusis is widely recognized, while there is no broad consensus as to the specific mechanisms or loci generating these hearing disorders. ${ }^{14}$ Many studies in both animals and humans have led to a number of proposed neurophysiological mechanisms, including tonotopic map reorganization, changes in spontaneous activity, altered neural synchrony along the auditory pathway or aberrant filtering of auditory information by limbic regions. ${ }^{14,15}$ Recently, in a study of Auerbach and Salvi, enhanced central gain in many auditory areas has been hypothesized to be a potential mechanism that gives rise to hyperacusis and tinnitus in response to a variety of acoustic or ototoxic insults. ${ }^{14}$

Ototoxic drugs, such as carboplatin and salicylate, can cause cochlear damage and some of them preferentially destroy specific structures in the cochlea. ${ }^{14}$ In another study, the authors suggested that high-dose long-term treatment with salicylate may damage the neurites, soma or axons of spiral ganglion neurons (SGN). ${ }^{16}$ Moreover, moderate and high stress levels at the time of acoustic trauma have been suggested to play a pivotal role in the vulnerability of the cochlea to acoustic damages and, therefore, for the development of tinnitus and hyperacusis. ${ }^{10}$

Hyperacusis is a subjective phenomenon, which is not easily defined or quantified by objective measurements. ${ }^{17}$ The determination of uncomfortable loudness levels (ULLs) is a common audiological test, used in the assessment of hyperacusis. ${ }^{18}$ However, the test methodology for its assessment varies and it is not standardized. Moreover, there is significant variability in the interpretation of test data, recommended for its identification. ${ }^{19}$ To satisfy the necessity to have an indirect tool suitable to quantify and evaluate hyperacusis, various questionnaires were created in order to screen several aspects of auditory symptomatology. A questionnaire largely used is the Khalfa's questionnaire on hyperacusis (HQ). ${ }^{17} \mathrm{~A}$ validated Dutch version of HQ is available $\mathrm{e}^{20}$ and a Japanese version of the HQ was recently developed, showing high reliability and validity. ${ }^{21}$

In a previous study, we validated the Italian version of the HQ in view of its use as an essential tool in the evaluation of hyperacusis symptomatology and we found a cut-off of 16 , indicative of hyperacusis instead of the previous cut-off of 28 identified by Khalfa to represent strong auditory hypersensitivity. The Italian version of HQ has an high and good internal consistency reliability (Cronbach $\alpha$ index $=0.89$, sensibility $=67.89 \%$, specificity $=$ $72.22 \%)^{22}$

The purpose of this study was to explore the collapsibility of the number of items of the Italian version of the HQ and to verify the possibility to use a reduced version of this tool, the short hyperacusis questionnaire (SHQ).

\section{Materials and Methods}

\section{Participants}

We recruited 117 consecutive outpatients, 68 male (58\%) and 49 female $(42 \%)$, age range $23-82$ years, $($ mean $=53$ years $)$, with a primary complaint of tinnitus at least from 3 months. All patients filled in the complete Italian version of the HQ and the tinnitus handicap inventory (THI) in a self-administered way. An accurate anamnesis was performed. Patients also underwent an ENT clinical examination with otoscopy, rhinoscopy and faringoscopy. The audiological examination was carried out into an audiological cabin, using an audiometer and included tonal audiometry, pitch and loudness tinnitus matching, ULLs (Table 1). Normal hearing was defined by threshold $<25 \mathrm{~dB}$ HL in all frequencies tested.

Exclusion criteria were the presence of loudness recruitment and Ménière's disease, evaluated with anamnesis and audiological data, as well as a previous diagnosis of psychiatric disease. Loudness recruitment describes an experience commonly associated with cochlear hearing loss and specifically with dysfunction of the outer hair cells of the organ of Corti: with a rising sound level, the perceived loudness increases faster than normal. We excluded patients with this auditory symptom because it could be confused with hyperacusis.

In a similar way, we excluded patients affected with Meniere's disease because a sensorineural hearing loss (SNHL) for the medium-low frequencies is often present in this pathology and the tonal audiometric curve can undergo some variations during recrudescence of the pathology. In our study, all the participants had normal hearing or a mild SNHL only for the high frequencies (6000 and $8000 \mathrm{~Hz}$ ).

Informed consent was obtained from each participant before examination. Nevertheless, every patient gave consent for the use of anonymous of course data, eventually for a future study. Since our study is an observational one and it was carried out using questionnaires and data of non-invasive almost daily routine exams, such as audiometry, it was not requested a formal medical approval by the local medical ethics committee.

\section{HQ}

The HQ is divided into 2 parts: the first part includes 3 binary questions giving general information on auditory disorders and noise exposure and the second part comprises 14 self-rating items. The answers to each question/item are given on a 4-point scale, ranging from no (scoring 0 points), yes, a little (scoring 1 point), yes, quite a lot (scoring 2 points) to yes, a lot (scoring 3 points). A statistical analysis performed on the principal components of the 14 items isolated three different dimensions: attentional (items from 1 to 4 inclusive), social (items from 5 to 10 inclusive) and emotional dimension (items from 11 to 14 inclusive). ${ }^{17}$

\section{ULLs}

ULLs were determined according to an accurate method. Each participant was instructed to let the examiner know when the pure tone became uncomfortably loud. The testing started at $1 \mathrm{kHz}$ with a signal intensity of $70 \mathrm{~dB}$. If this level was perceived as

Table 1. Demographic and audiological variables for patients.

\begin{tabular}{lcc} 
Gender & Male & Female \\
& 68 & 49 \\
Audiological exam & NH & SNHL \\
\hline ULLs & 47 & 70 \\
& Normal & Altered \\
\hline
\end{tabular}

NH, normal hearing; SNHL, sensorineural hearing loss; ULLs, uncomfortable loudness levels. 
uncomfortably loud, the intensity was decreased by $10 \mathrm{~dB}$ and the starting level on the following frequencies was decreased to $60 \mathrm{~dB}$. The sound intensity was increased in $5 \mathrm{~dB}$ steps until the participant gave a response that the sound was uncomfortably loud. The tested frequencies were $0.25,0.5,1,2,4$ and $8 \mathrm{kHz}$.

All ULLs' tests were done giving exact instructions as recommended by the British Society of Audiology (BSA, 2011): I will gradually make the sound louder in your ear, and you must raise your hand as soon as the sound becomes uncomfortably loud. This is not a test to find the loudest sound you can tolerate; it is a test to find what level of sound you find uncomfortable. You should raise your hand only when the sound becomes uncomfortable; but make sure you raise it as soon as the sound reaches that level. ${ }^{23}$ Mild hyperacusis was considered in presence of ULLs at 80-90 dB in 2 or more frequencies, moderate hyperacusis in presence of ULLs at $65-75 \mathrm{~dB}$ in 2 or more frequencies and severe hyperacusis in presence of ULLs at $60 \mathrm{~dB}$ or lower in 2 or more frequencies (Table 2). ${ }^{19}$

\section{Statistical analysis}

The analysis provided descriptive statistics for the questionnaire items. A survey of the frequency distribution of the ULLs variable showed that the modalities moderate and severe occurred just four times (3.41\%) [three patients with moderate ULLs (2.56\%), only one with severe ULLs $(0.85 \%)$ ] on 117 patients (Table 3, Figure 1). This feature suggested collapsing the ULLs just on the normal and mild modalities. This choice changed the problem from a multinomial structure to a binomial one, so that we could use a logistic model, simplifying both the analysis and the clinical interpretation of the results. Therefore, we excluded four participants from the study obtaining a final sample of 113 patients (Table 4).

In order to explore the collapsibility of the questionnaire number of items, a logistic model has been carried out getting odds ratios (ORs) estimates of hyperacusis according to the items responses. The resulting items' ORs statistical significance was exploited to select the items with statistically significant values different from 1. According to this procedure, six items were selected: one item coming from the attentional dimension, four items from the social dimension and one from the emotional dimension. The overall fitting was assessed using LR test and Wald test for each item's covariate.

The discriminant role played by patients' gender has been previously tested using Hotelling multivariate test and it did not reach a statistical significant value $(\mathrm{P}>0.05)$. This test was performed both for the complete set of the questionnaire items and for the six ones selected. So that no gender adjustment was required for the logistic regression.

Internal consistency of the selected items was assessed by means of Cronbach $\alpha$ index and a non-parametric ROC analysis was performed using the corresponding estimated logistic scoring function. The overall performance has been described by the area under the ROC (AUC). Optimal cut-off point and the corresponding test sensitivity and specificity were found using the Youden index. Because of the use of a logit model, test scores are probability values less than 1 .

The statistical analysis was carried out using the statistical software STATA version 14.

\section{Results}

Based on the ULLs, we found 38 (33.63\%) participants with mild hyperacusis: 9 patients $(7.96 \%)$ with normal hearing and 29 patients $(25.66 \%)$ with high sensorineural hearing loss.

From the first logistic model, the odds estimates resulted statically significant for item 3 , item 6 , item 7 , item 8 , item 9 and item 12 of the HQ $(\mathrm{P}<0.05)$. These remarks showed the dominance of the following items: item 3 in predicting the hyperacusis status for the attentional dimension, items $6,7,8$ and 9 in predicting the hyperacusis status for the social dimension and item 12 for the

Table 2. Audiological classification of hyperacusis based on the ULLs findings.

\begin{tabular}{ll} 
ULL (dB HL) & Hyperacusis \\
$>90 \mathrm{~dB}$ in all frequencies & Absence of hyperacusis \\
$80-90 \mathrm{~dB}$ in 2 or more frequencies & Mild hyperacusis \\
\hline $65-75 \mathrm{~dB}$ in 2 or more frequencies & Moderate hyperacusis \\
$<65 \mathrm{~dB}$ in 2 or more frequencies & Severe hyperacusis \\
\hline
\end{tabular}

Modified from Goldstein et al., $1996 .{ }^{19}$

Table 3. Distribution of patients with mild, moderate, severe hyperacusis based on ULLs data.

\begin{tabular}{lcc} 
ULLs & Patients (n) & Percentage (\%) \\
Normal & 75 & 64.10 \\
Mild & 38 & 32.48 \\
\hline Moderate & 3 & 2.56 \\
Severe & 1 & 0.85 \\
\hline Total & 117 & 100.00 \\
\hline
\end{tabular}

Table 4. Distribution of normal patients and patients with mild hyperacusis based on ULLs after dropping patients with moderate and severe hyperacusis based on ULLs.

\begin{tabular}{lcc} 
ULLLs & Patients (n) & Percentage (\%) \\
Normal & 75 & 66.37 \\
Mild & 38 & 33.63 \\
\hline Total & 113 & 100.00 \\
\hline
\end{tabular}

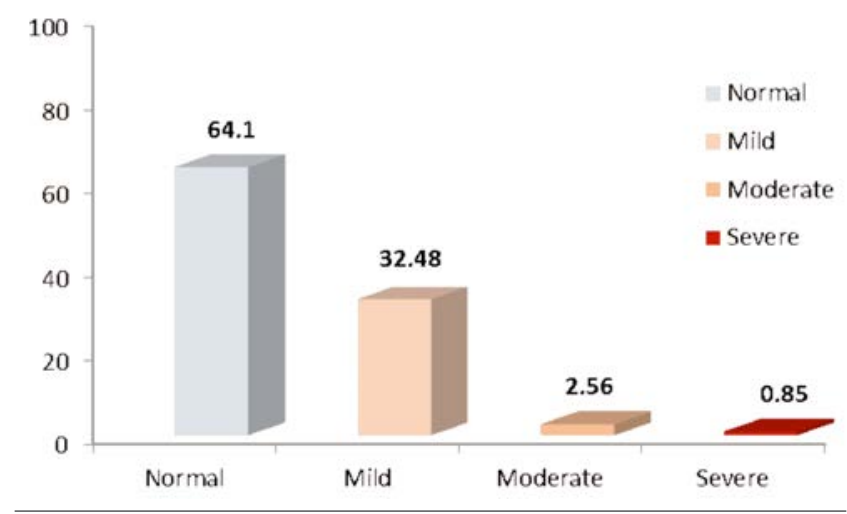

Figure 1. Distribution of patients in percentage (\%) based on ULLs data. 
emotional dimension (Table 5).

Our analysis of the SHQ, using the selected items (3, 6, 7, 8, 9 and 12) showed a Cronbach $\alpha$ index of 0.67 with a scale reliability less statistically significant compared to that of the HQ (Cronbach $\alpha$ index $=0.89$ ). In order to perform a ROC analysis we estimated a logistic scoring function, which provided a corresponding score for each items' values.

The area under the ROC curve of hyperacusis (AUC = $0.67 \pm 0.05)$, expression of the overall performance of the SHQ versus the ULLs test was statistically significant $(\mathrm{P}<0.05)$ (Figure 2). Moreover, we found a cut-off of 0.24 as indicative of hyperacusis (sensitivity $(\mathrm{Se})=78.79 \%$, specificity $(\mathrm{Sp})=42.50 \%)$ by performing a ROC analysis, conditioned to patients affected with tinnitus, and by using ULLs as reference level. In our study, 72 patients with tinnitus ( $64 \%$ of the total of participants who performed the SHQ) obtained a total score indicative of hyperacusis.

\section{Discussion}

In previous studies, the hyperacusis was found in the $40-45 \%$ of individuals with tinnitus. ${ }^{24}$ The difference of prevalence of hyperacusis found by many authors may depend by the criteria used to define hyperacusis. There is no universally agreed definition of hyperacusis. Audiologists often use ULLs to diagnose hyperacusis evaluating the lowest sound level at which sounds are perceived to be uncomfortable or painful. However, the ULLs assessment is variable and not standardized with a significant variability in the interpretation of test data among different studies. ${ }^{19}$ Anyway, some human studies indicate that auditory deprivation can only decrease ULLs by $7 \mathrm{~dB}$ relative to their normal levels. ${ }^{25}$ This is not consistent with the 40-50 dB shift in ULLs, which is common among patients with hyperacusis. ${ }^{4}$ Therefore, the use of ULLs in defining hyperacusis patient is debated. Meeus reported no differences in the ULL scores between hyperacusis and non-hyperacusis patients, and no correlations between the ULLs and HQ scores suggesting incongruences between audiometric measurements and hyperacusis complaints as noted by Sammeth. ${ }^{26}$

The necessity to have an indirect tool, suitable to quantify and evaluate hyperacusis, has led to the creation of various questionnaires in order to screen several aspects of this auditory symptom. The hyperacusis questionnaire (HQ) is only one of them and it used by clinicians for the quantification and characterization of the clinical phenomenon of hyperacusis over three major dimensions: attentional, social and emotional. ${ }^{17}$ Anyways, the criteria for diagnosing hyperacusis handicap based on the HQ are also not generally agreed. For example, Khalfa et al. ${ }^{17}$ suggested a cut-off score of 28 as indicative of severe hyperacusis, while
Fackrell et al. ${ }^{27}$ suggested reevaluating the cut-off score of 28 . In the validation of the HQ for the Italian population, we suggested a cut-off of 16 indicative of hyperacusis. ${ }^{22}$ Other questionnaires such as the test of hypersensitivity to sound (THS/GÜF) 28 and the multiple-activity scale for hyperacusis $(\mathrm{MASH})^{2}$ are also available to assess the subjective distress related to the hypersensitivity to sound.

Recently, it was shown by Schecklemann that also using only two questions of the tinnitus sample case history (TSCH) can give a good hint at whether hyperacusis is present: i) Do you have a problem tolerating sounds because they often seem much too loud? ii) Do sounds cause you pain or physical discomfort? In fact, in this study, $55 \%$ out of 1713 patients with tinnitus were characterized as hyperacusis patients. ${ }^{29}$

Furthermore, Fackrell suggested that only ten items and two dimensions (attentional and social) of the HQ might be a more appropriate approach for assessing hyperacusis instead of the current fourteen items and three dimensions (attentional, social and emotional). ${ }^{27}$ Comparing these results, there are some similarities in the selection of the most significant questions. However, in our case, it remains the presence of all three dimensions and this can be an advantage, as we believe that all of them have a diagnostic significance. In fact, hyperacusis is a multidimensional phenomenon and so co-morbidities need to be taken into account. Several studies highlight the importance of assessing emotional exhaustion in the diagnosis of hyperacusis, particularly in women, even if the underlying reasons for the sex-related differences are not completely clear yet. Moreover, the same studies also underline the need to

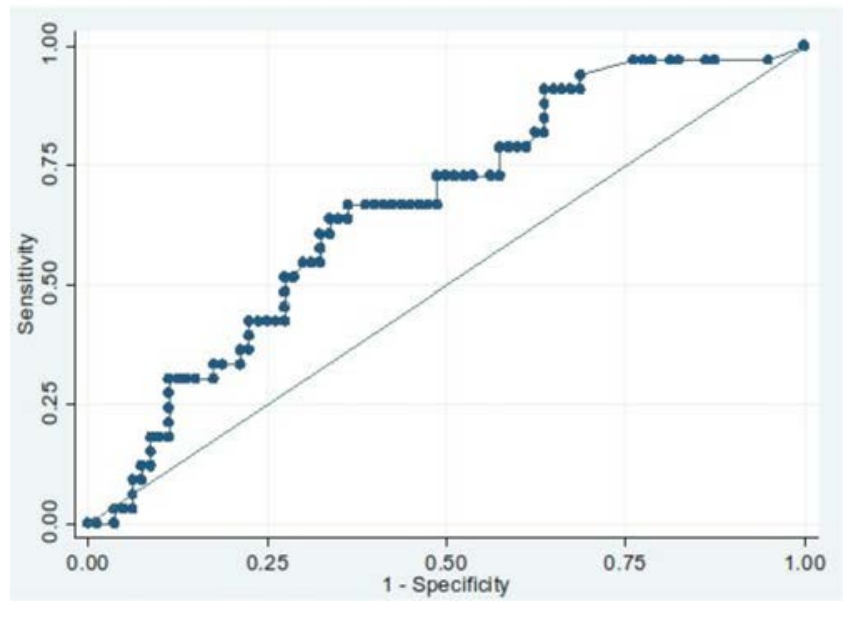

Figure 2. ROC analysis for the logit scores of SHQ using mild ULLs as reference level (AUC $=0.67 \pm 0.05$ ).

Table 5. Odd ratios (ORs) and P-value of the six selected items for the SHQ.

\begin{tabular}{|c|c|c|c|}
\hline Dimensions & Items & OR & P-value \\
\hline Attentional & Item 3: Do you have trouble reading in a noisy or loud environment? & 1.08 & 0.03 \\
\hline Social & Item 6: Has anyone you know ever told you that you tolerate noise or certain kinds of sound badly? & 1.17 & 0.03 \\
\hline Social & Item 7: Are you particularly sensitive to or bothered by street noise? & 1.16 & 0.04 \\
\hline Social & $\begin{array}{l}\text { Item 8: Do you find the noise unpleasant in certain social situations } \\
\text { (e.g. night clubs, pubs or bars, concerts, firework displays, cocktail receptions)? }\end{array}$ & 1.24 & 0.04 \\
\hline Social & $\begin{array}{l}\text { Item 9: When someone suggests doing something (going out, to the cinema, to a concert, etc.), } \\
\text { do you immediately think about the noise you are going to have to put up with? }\end{array}$ & 1.25 & 0.04 \\
\hline Emotional & Item 12: Do stress and tiredness reduce your ability to concentrate in noise? & 1.06 & 0.04 \\
\hline
\end{tabular}


include stress management for an effective treatment of hyperacusis. ${ }^{10,18}$

In our analysis, the SHQ enables to investigate which dimension is the most affected by the pathology, due to the persistence of the distribution of items in the three dimensions. Anyways, we have to remark that the SHQ shows a dominance of the social dimension's items (item 6, 7, 8 and 9). In fact, the ORs of the item 3 and item 12 ( 1.08 and 1.06 respectively) can be questionable but the two items in questions represent both the only item statistically significant, characterizing a dimension (items 3 for the attentional dimension and item 12 for the emotional dimension) and, therefore, we consider them clinically relevant for the hyperacusis assessment.

On the contrary, the SHQ lacks in internal consistency, with a Cronbach $\alpha$ of 0.67 less statistically significant compared to that of the Italian version of the HQ (Cronbach $\alpha=0.89$ ), ${ }^{22}$ allowing a poorer performance in the identification of the individuals who suffer from this condition. In fact, the overall performance of the SHQ was less satisfactory of the Italian version of $\mathrm{HQ}$, as described by the AUC of $0.67 \pm 0.05(\mathrm{P}<0.05)$. On the contrary, we think that the cut-off of 0.24 , as indicative of hyperacusis, presents a good sensitivity and specificity for a screening questionnaire $(\mathrm{Se}=$ $78.79 \%, \mathrm{Sp}=42.50 \%$ ). According to this cut-off, $64 \%$ of participants of our study had hyperacusis.

Lastly, we have to consider some issues as limitations of our study. Auditory damage, as hidden hearing loss, could escape tonal audiometry and in these cases speech audiometry and auditory brain stem response (ABR) could add important details. In the audiological assessment, we only determined the ULLs for tones while we did not determinate the ULLs for narrowband or broadband noises even if most of the stimuli in natural setting are not tonal. The size of our sample was not very large (113 patients) and limited to consecutive patients with tinnitus but not to patients with only hyperacusis. This selection bias prevented us from investigating the presence of hyperacusis in the general population. Moreover, the cut-off, so identified of the SHQ, is an expression of a mostly dichotomous distinction between subjects with and without hyperacusis but it does not make a distintion of mild, moderate and severe pathology.

\section{Conclusions}

In our study, we tried to verify if the SHQ could be useful in the initial screening of individuals with hyperacusis. The main goal of our study was to provide Italian clinicians and researchers with a validated tool that could facilitate the identification of hyperacusis. In fact, we supposed that this new tool could be quite useful when we have many questionnaires to administer to the same patient and we want to investigate also hyperacusis in the large spectrum of the auditory symptoms, in particular, when hyperacusis is the prevailing disorder.

However, the SHQ showed a Cronbach $\alpha$ with a scale reliability less statistically significant compared to that of the Italian version of the HQ and a cut-off with a sensitivity of $78.79 \%$ and specificity of $42.50 \%$, conditioned to patients affected with tinnitus. Therefore, it could be used only for an initial screening of patients but not as the main questionnaire for the assessment and the quantification of hyperacusis.

In alternative to the SHQ, we can consider the possibility to formulate and propose a new questionnaire, which will consist of questions, previously selected for their internal consistency, articulated in the most appropriate manner and without the possibility of generating confusion in the patient. To facilitate this, we think it would be most appropriate for each country to develop its own questionnaire, based on the uses and the customs of the society. Moreover, patients seeking help for hyperacusis, but who exhibit normal ULLs, should also be assessed for emotional exhaustion for a correct diagnosis. We suggest further studies for the validation of a new questionnaire on hyperacusis.

\section{References}

1. Gu JW, Halpin CF, Melcher JR. Tinnitus, diminished soundlevel tolerance and elevated auditory activity in humans with clinically normal hearing sensitivity. J Neurophysiol 2010;104: 3361-70.

2. Dauman R, Bouscau-Faure F. Assessment and amelioration of hyperacusis in tinnitus patients. Acta Otolaryngol 2005;125: 503-9.

3. Andersson G, Lindvall N, Carlbring P. Hypersensitivity to sound (hyperacusis): a prevalence study conducted via the Internet and post. Int J Audiol 2002;41:545-54.

4. Aazh H, McFerran D, Jastreboff P. Insights from the first international conference on hyperacusis: causes, evaluation, diagnosis and treatment. Noise Health 2014;16:123-6.

5. Jastreboff PJ, Jastreboff MM. Decreased sound tolerance. In: Snow JB Jr, ed. Tinnitus: theory and management. Ch. 2. London, Hamilton: BC Decker; 2004. pp 8-15.

6. Tyler RS, Noble W, Bardia A. Tinnitus and hyperacusis. In: Katz J, Burkard R, Medwetsky L, Hood L, eds. Handbook of clinical audiology. $6^{\text {th }}$ ed. Baltimore: Lippincott Williams and Wilkins; 2009.

7. Molander P, Nordqvist P, Andersson G. Internetbased hearing screening using speech-in-noise: validation and comparisons of self-reported hearing problems, quality of life and phonological representation. BMJ Open 2013;16.

8 Chen G, Lee C, Kaltenbach JA. Behavioral evidence for possible simultaneous induction of hyperacusis and tinnitus following intense sound exposure. J Assoc Res Otolaryngol 2013;14:413-24.

9. Heeringa AN, van Dijk P. The dissimilar time course of temporary threshold shifts and reduction of inhibition in the inferior colliculus following intense sound exposure. Hear Res 2014;312:38-47.

10. Knipper M, Van Dijk P, Zimmermann U. Advances in the neurobiology of hearing disorders: recent developments regarding the basis of tinnitus and hyperacusis. Prog Neurobiol 2013;111:17-33.

11. Schaette R, McAlpine D. Tinnitus with a normal audiogram: physiological evidence for hidden hearing loss and computational model. J Neurosci 2011;31:13452-7.

12. Weisz N, Hartmann T, Norena A. High-frequency tinnitus without hearing loss does not mean absence of deafferentation. Hear Res 2006;222:108-14.

13. Liberman MC, Kujawa SG. Cochlear synaptopathy in acquired sensorineural hearing loss: Manifestations and mechanisms. Hear Res 2017;349:138-47.

14. Auerbach BD, Rodrigues PV, Salvi RJ Central gain control in tinnitus and hyperacusis. Front Neurol 2014;5:206.

15. Hayes SH, Radziwon KE, Stolzberg DJ, Salvi RJ. Behavioral models of tinnitus and hyperacusis in animals. Front Neurol 2014;5:179.

16. Chen GD, Kermany MH, Salvi R. Too much of a good thing: long-term treatment with salicylate strengthens outer hair cell function but impairs auditory neural activity. Hear Res 2010;265:63-9. 
17. Khalfa S, Dubal S, Jouvent R, et al. Psychometric normalization of a hyperacusis questionnaire. ORL J Otorhinolaryngol Relat Spec 2002;64:436-42.

18. Hasson D, Theorell T, Bergquist J, Canlon B. Acute stress induces hyperacusis in women with high levels of emotional exhaustion. PLoS One 2013;8:e52945.

19. Goldstein B, Shulman A. Tinnitus - hyperacusis and the loudness discomfort level test - a preliminary report. Int Tinnitus J 1996;2:83-9.

20. Meeus OM, Spaepen M, Ridder DD, Heyning PH. Correlation between hyperacusis measurements in daily ENT practice. Int J Audiol 2010;49:7-13.

21. Oishi N, Yamada H, Ogawa K. Assessment of hyperacusis with a newly produced Japanese version of the Khalfa hyperacusis questionnaire. Acta Otolaryngol 2017;1-5.

22. Fioretti A, Tortorella F, Pavaci S. Validity of the Italian version of Khalfa's questionnaire on hyperacusis. Acta Otorhinolaryngol Ital 2015;35:110-5.

23. Aazh H, Moore BCJ. Factors related to uncomfortable loudness levels for patients seen in a tinnitus and hyperacusis clinic. Int J Audiol 2017;16:1-8.
24. Jastreboff PJ, Jastreboff MM. Tinnitus retraining therapy for patients with tinnitus and decreased sound tolerance. Otolaryngol Clin North Am 2003;36:321-36.

25. Munro KJ, Blount J. Adaptive plasticity in brainstem of adult listeners following earplug-induced deprivation. J Acoust Soc Am 2009;126:568-71.

26. Sammeth CA, Preves DA, Brandy WT. Hyperacusis: case studies and evaluation of electronic loudness suppression devices as a treatment approach. Scand Audiol 2000;29:28-36.

27. Fackrell K, Fearnley C, Hoare DJ, Sereda M. Hyperacusis questionnaire as a tool for measuring hypersensitivity to sound in a tinnitus research population. Biomed Res Int 2015;2015:290425.

28. Nelting M, Rienhoff NK, Hesse G, Lamparter U. The assessment of subjective distress related to hyperacusis with a self-rating questionnaire on hypersensitivity to sound. Laryngorhinootologie 2002;81:327-34.

29. Schecklmann M, Landgrebe M, Langguth B; TRI database study group. Phenotypic characteristics of hyperacusis in tinnitus. PLoS One 2014;9:e86944. 